\title{
Risk Factors for Hospital-Acquired Pneumonia in Nonventilated Adults
}

\author{
Carlos Magno Castelo Branco Fortaleza ${ }^{1,2}$, Paulo Afonso Martins Abati ${ }^{1}$, Márcia Regina Batista ${ }^{2}$ and Adriano Dias ${ }^{3}$ \\ ${ }^{1}$ Departamento de Doenças Tropicais, Faculdade de Medicina de Botucatu, UNESP - Universidade Estadual Paulista. Botucatu, SP; ${ }^{2}$ Hospital \\ Estadual Bauru, Faculdade de Medicina de Botucatu, UNESP - Universidade Estadual Paulista. Bauru, SP; ${ }^{3}$ Departamento de Saúde Pública, \\ Faculdade de Medicina de Botucatu, UNESP - Universidade Estadual Paulista, Botucatu, SP; Brazil
}

\begin{abstract}
Although most recent publications focus on Ventilator-associated Pneumonia, Non-Ventilator-associated Hospitalacquired pneumonia (NVHAP) is still worrisome. We studied risk factors for NVHAP among patients admitted to a small teaching hospital. Sixty-six NVHAP case patients and 66 controls admitted to the hospital from November 2005 through November 2006 were enrolled in a case-control study. Variables under investigation included: demographic characteristics, comorbidities, procedures, invasive devices and use of medications (Sedatives, Antacids, Steroids and Antimicrobials). Univariate and multivariable analysis (hierarchical models of logistic regression) were performed. The incidence of NVHAP in our hospital was $0.68 \%$ (1.02 per 1,000 patients-day). Results from multivariable analysis identified risk factors for NVHAP: age (Odds Ratio[OR]=1.03, 95\% Confidence Interval $[C I]=1.01-1.05, p=0.002)$, use of Antacids $(O R=5.29,95 \% C I=1.89-4.79, p=0.001)$ and Central Nervous System disease $(\mathrm{OR}=3.13,95 \% \mathrm{CI}=1.24-7.93, \mathrm{p}=0.02)$. Although our findings are coherent with previous reports, the association of Antacids with NVHAP recalls a controversial issue in the physiopathology of Hospital-Acquired Pneumonia, with possible implications for preventive strategies.
\end{abstract}

Key-Words: Hospital acquired pneumonia, non-ventilator associated pneumonia, risk factors.

Despite current emphasis in the control of ventilatorassociated pneumonia (VAP), hospital or healthcare-acquired pneumonia in patients that were not submitted to mechanical ventilation is still a matter of concern. It is still common among patients with chronic diseases, immune suppressing conditions, or who are submitted to surgical procedures [1,2]. It is also specially incident in elders, either in acute care settings or in nursing homes $[3,4]$. In all those cases, it has significant impact on morbidity and mortality rates.

Previous studies report a wide variety of risk factors for Non-Ventilator Associated Hospital-Acquired Pneumonia (NVHAP). Their results vary according to the population from which cases are selected. For instance, risk factors among patients who were not submitted to surgical procedures differ from those reported for post-operative NVHAP [5,6]. Our study was designed to identify risk factors for NVHAP among adult patients admitted to a general teaching hospital.

\section{Material and Methods}

Setting

This study was conducted in Hospital Estadual Bauru, one of the teaching hospitals from Faculdade de Medicina de Botucatu (Botucatu School of Medicine). The hospital has 280 active beds, four intensive care units, five wards for adult patients and one pediatric unit. Active hospital-wide surveillance of Hospital-acquired Infections is routinely performed by the Infection Control Committee (ICC).

Received on 10 January 2009; revised 16 June 2009.

Address for correspondence: Dr. Carlos Magno Castelo Branco Fortaleza, Departamento de Doenças Tropicais, Faculdade de Medicina de Botucatu. Distrito de Rubião Júnior, Botucatu, São Paulo State, Brazil Zip code: 18618-970. Email: cmfortaleza@uol.com.br. Phone: 5514 38116212 - FAX: 551438159898.

The Brazilian Journal of Infectious Diseases 2009;13(4):284-288. (C) 2009 by The Brazilian Journal of Infectious Diseases and Contexto Publishing. All rights reserved.
Study Design

The study had a case-control design. Both cases and controls were selected from adult patients admitted to the hospital from November 2005 through November 2006.

\section{Case Definition and Control Selection}

We searched ICC files to find patients with diagnosis of NVHAP. All cases were reviewed for meeting current Centers for Diseases Control and Prevention (CDC) criteria for diagnosis of nosocomial pneumonia. Patients were enrolled as cases if they met the "PNU1" CDC criteria [7]. For each case, we selected a control that was admitted in the same unit at the same time as the case patient.

Exclusion criteria for both cases and controls were: hospital stay of less than 48 hours; age under 18; report of mechanical ventilation during this admission or in the previous year. Patients submitted to surgery were enrolled in the study if they had not been on mechanical ventilation for a longer period than was strictly necessary for the performance of the surgical procedure.

\section{Investigation of Risk Factors}

Patient data were recorded from medical charts and laboratory database. Definitions of underlying conditions followed the guidelines from International Classification of Diseases, tenth edition [8]. The burden of comorbid conditions was assessed using the Charlson comorbidity Index [9]. Nutritional status and previous history of smoking, alcoholism (defined as self report of daily consumption of alcoholic beverages) and illicit drug use were recorded. Other data were analyzed from the day of hospital admission until the day of NVHAP diagnosis (for case patients) or until discharge (for control subjects). Data included a present or previous intensive care unit (ICU) stay; performance of surgery; restriction to the bed and use of devices. From medical prescriptions, we recorded the use of Antacids 
(Ranitidine or Omeprazole), Sedatives, Steroids (or other immune-suppressing drugs) and Antimicrobials.

To assess the time of exposure to hospital environment, we used a variable termed "time at risk". For case patients, "time at risk" was defined as the number of days from admission to our hospital up to the diagnosis of NVHAP; for control subjects, it corresponded to the total number of days of hospitalization.

\section{Statistical Analysis}

Data were recorded in Epi Info Software for Windows, version 3.2 (@Centers for Disease Control and Prevention) and analyzed with SPSS software, version 15.0 (CSPSS Inc.). Each variable was submitted to univariate analysis. Dichotomous variables were analyzed using the Chi-square or Fisher's Exact Test. For numeric variables we used Student's T test or the Mann-Whitney test. All variables for which $\mathrm{p}<0.2$ were included in hierarchical multiple logistic regression model [10]. We used a stepwise forward approach, setting $p=0.05$ as the limit for acceptance or removal of the terms in the model. Groups of variables entered the hierarchical model in the following order: (1) demographic variables; (2) comorbidities; (3) therapeutic interventions during hospital admission, including procedures, invasive devices and drugs (except antimicrobials); and (4) previous use of antimicrobials. A pvalue of less than 0.05 set as the final limit for significance.

\section{Definition of Etiology}

The definition of NVHAP etiology was not the main objective of our study. Given the retrospective nature of the study, it was not possible to standardize the investigation of etiologic agents. However, most patients had blood cultures collected during NVHAP investigation, and a few of them had also been submitted to bronchoscopic procedures. For etiological purposes, we considered valid the microbiological results from blood cultures or bronchoalveolar lavage (BAL) specimens collected during investigation of the present NVHAP episode, or in the first 48 hours after its clinical diagnosis. All cultures from BAL specimens were quantitative, and only growth above $10^{4}$ colony forming units $/ \mathrm{mL}$ was considered significant. Single blood cultures that were positive for coagulase-negative staphylococci were excluded from analysis. Antimicrobial susceptibility tests were performed according to current standards [11].

\section{Ethical Issues}

This study was submitted to the Cientific Committee of Hospital Estadual Bauru and to the reference Research Ethics Committee (Instituto Lauro de Souza Lima, Bauru City, Brazil), and obtained full approval.

\section{Results}

In the study period, 9,641 adult patients were admitted to the hospital. Sixty-six patients met diagnostic criteria for NVHAP. The incidence rate was $0.68 \%$ (or 1.02 per 1,000 patients-day).

The case patients were enrolled in the case-control study along with an equal number of control subjects. Several risk factors were identified in univariate analysis: age, comorbidites (Renal and Central Nervous System [CNS] diseases) and invasive devices (Central Venous Catheter, Urinary Catheter, Naso-enteral tube). The use of 3rd/4th generation Cephalosporins or Vancomycin were negatively associated with NVHAP incidence in the univariate analysis. Full results are presented in Table 1.

In multivariable logistic regression model (Table 2), independent risk factors for NVHAP were: age (Odds Ratio[OR $]=1.03$, 95\% Confidence Interval[CI]=1.01-1.05, $\mathrm{p}=0.002)$, use of Antacids (OR=5.29, 95\%CI=1.89-4.79, $\mathrm{p}=0.001)$ and Central Nervous System disease (OR=3.13, 95\%CI=1.247.93, $\mathrm{p}=0.02$ ).

Results from blood cultures were available for 58 patients. Other 13 cases collected cultures from BAL. However, the identification of the etiological agent was made in only seven case patients (10.6\%). Five of them had cultures positive for multidrug-susceptible bacterial strains (Table 3).

\section{Discussion}

Mechanical ventilation was identified as a primary risk factor for hospital-acquired pneumonia in studies from the 1980s [5,12]. Since then, VAP has been specifically addressed as a condition which poses a major threat for hospitalized patients $[13,14]$. Therefore, while many studies have focused on risk factors and prevention measures for VAP, few recent publications address the epidemiology of NVHAP $[13,14]$. According to Sopena et al, two reasons account for the small number of studies addressing NVHAP: (1) the great dispersion of NVHAP cases within hospitals, which hinders surveillance; (2) the difficulty of performing invasive diagnostic techniques in this population [15]. We must add that the lack of uniform diagnostic criteria in the literature reduces the possibility of comparing data from different authors.

Risk factors commonly reported for hospital-acquired pneumonia often fall into one of the following cathegories: (1) factors that enhance colonization of the oropharinx or stomach; (2) conditions that favor aspiration into the respiratory tract; (3) prolonged ventilatory support; and (4) host factors, such as extremes of age and severe underlying conditions [14]. The findings in our study are coherent with those cathegories.

Age was a significant risk factor for NVHAP. Some authors attribute the greater incidence of pneumonia among elderly people to the presence of comorbid illnesses or to longer hospital stay, rather than for chronological age "per se" [14,16]. However, we have studied the impact of age in multivariable models that included several confounders (comorbidities, time at risk, severity-of-illness measured by the Charlson index) and it still remained an independent risk factor for NVHAP. This may be due to several physiological and immunological changes that are inherent to aging process. 
Table 1. Results of univariate analysis of risk factors for non-ventilator associated hospital acquired-pneumonia

\begin{tabular}{|c|c|c|c|c|}
\hline Risk factors & Cases $(n=66)$ & Controls $(n=66)$ & OR $(95 \% C I)$ & $\mathbf{p}$ \\
\hline \multicolumn{5}{|l|}{ Demographic data } \\
\hline Female Sex & $29(43.9)$ & $21(31.8)$ & $1.68(0.83-3.42)$ & 0.15 \\
\hline Age, mean (median) & $70.7(73)$ & 57.4(59.5) & & $<0.001 \mathrm{a}$ \\
\hline \multicolumn{5}{|l|}{ Comorbidities } \\
\hline Cardiac disease & $23(34.8)$ & $20(30.6)$ & $1.23(0.59-2.55)$ & 0.58 \\
\hline Pulmonary disease & $11(16.7)$ & $15(22.7)$ & $0.68(0.29-1.62)$ & 0.38 \\
\hline Renal disease & $9(13.6)$ & 0 & $\ldots$ & $0.001 \mathrm{a}$ \\
\hline Liver disease & $4(6.1)$ & $1(1.5)$ & $4.19(0.45-38.57)$ & 0.18 \\
\hline CNS disease & $33(50.0)$ & $16(24.2)$ & $3.13(1.46-6.56)$ & $0.002 \mathrm{a}$ \\
\hline Diabetes mellitus & 19(28.8) & $12(18.2)$ & $1.82(0.80-4.14)$ & 0.15 \\
\hline Solid malignancy & $4(6.1)$ & $7(10.8)$ & $0.53(0.14-1.92)$ & 0.33 \\
\hline Aids & $1(1.5)$ & 0 & $\ldots$ & 0.5 \\
\hline Charlson comorbidity index, mean (median) & $2.7(2)$ & $2.2(2)$ & & 0.06 \\
\hline Poor nutritional status & $8(12.1)$ & $3(4.5)$ & $2.90(0.73-11.44)$ & 0.12 \\
\hline Smoking & $22(33.3)$ & $16(24.2)$ & $1.56(0.73-3.34)$ & 0.25 \\
\hline Alcoholism & $12(18.2)$ & $5(7.6)$ & $2.71(0.90-8.19)$ & 0.07 \\
\hline Use of illicit drugs & $1(1.5)$ & $1(1.5)$ & $1.00(0.06-16.33)$ & 0.75 \\
\hline \multicolumn{5}{|l|}{ Admission data } \\
\hline Time at risk, mean (median) & 16.6(13) & $18.0(13)$ & & 0.94 \\
\hline Present or previous ICU stay & $4(6.1)$ & 0 & $\ldots$ & 0.06 \\
\hline Neutropenia & 0 & $2(3.0)$ & $\ldots$ & 0.24 \\
\hline Use of steroids & $8(12.3)$ & $14(21.2)$ & $0.52(0.20-1.34)$ & 0.17 \\
\hline Use of other immunossupressive drugs & $2(3.0)$ & $4(6.1)$ & $0.48(0.08-2.74)$ & 0.34 \\
\hline Use of sedatives & $22(33.3)$ & $10(22.7)$ & $1.70(0.79-3.67)$ & 0.17 \\
\hline Use of antacids & $30(45.5)$ & $10(15.2)$ & $4.67(2.04-6.69)$ & $<0.001 \mathrm{a}$ \\
\hline Restriction to bed & $11(16.7)$ & $8(12.3)$ & $1.43(0.53-3.81)$ & 0.48 \\
\hline Surgery & $7(10.6)$ & $12(18.2)$ & $0.53(0.19-4.90)$ & 0.21 \\
\hline Central venous catheter & $13(19.7)$ & $5(7.6)$ & $2.99(1.00-8.95)$ & $0.04 a$ \\
\hline Urinary catheter & $25(37.9)$ & $14(21.2)$ & $2.26(1.05-4.90)$ & $0.04 \mathrm{a}$ \\
\hline Enteral tube feeding & $27(40.9)$ & $7(10.6)$ & $5.84(2.32-14.75)$ & $<0.001 \mathrm{a}$ \\
\hline Nasogastric tube drainage & $5(7.6)$ & $2(2.0)$ & $2.62(0.49-14.03)$ & 0.22 \\
\hline Parenteral nutrition & $1(1.5)$ & $1(1.5)$ & $1.00(0.06-16.33)$ & 0.75 \\
\hline Pressure ulcer & $9(13.6)$ & $4(6.2)$ & $2.41(0.70-8.25)$ & 0.15 \\
\hline \multicolumn{5}{|l|}{ Previous antimicrobial use } \\
\hline Penicillins & $4(6.1)$ & $10(15.2)$ & $0.26(0.11-1.22)$ & 0.09 \\
\hline Penicillins plus beta-lactamase inhibitors & 13(18.7) & $9(13.6)$ & $1.55(0.61-3.93)$ & 0.35 \\
\hline Cefalosporins, 1st generation & 0 & $4(6.1)$ & $0(\ldots)$ & 0.06 \\
\hline Cefalosporins, 3rd/4th generation & 0 & $5(7.6)$ & $0(\ldots)$ & $0.03 a$ \\
\hline Carbapenems & $1(1.5)$ & $1(1.5)$ & $1.00(0.06-16.33)$ & 0.75 \\
\hline Glicopeptides & 0 & $5(7.6)$ & $0(\ldots)$ & $0.03 a$ \\
\hline Aminoglicosides & 0 & $1(1.5)$ & $0(\ldots)$ & 0.55 \\
\hline Quinolones & $23(34.8)$ & $23(34.8)$ & $1(0.49-2.05)$ & 1.00 \\
\hline Clindamycin & 13(19.7) & $11(16.7)$ & $1.23(0.51-2.98)$ & 0.65 \\
\hline
\end{tabular}

Data are in no(\%), unless otherwise specified. Aids, acquired immunodeficiency syndrome; CI, confidence interval; CNS, central nervous system; ICU, intensive care unit; OR, odds ratio. a Statistically significant.

CNS diseases may depress cough reflexes, impair swallowing mechanisms and affect respiratory patterns [17]. All these alterations facilitate the access of microorganisms to the lower airways. Therefore, it was not surprising that CNS diseases were significant risk factors for NVHAP in our study.
The most puzzling finding in our study was the strong association between use of Antacids and increased risk for NVHAP. There are several papers on the role of Antacids in the physiopathology of VAP. It has been stated that alkalinization of the stomach provides an ideal environment for bacterial overgrowth - and, subsequently, to contamination 
Table 2. Results of multivariable analysis (hierarchical model of logistic regression) of risk factors for non-ventilator associated hospital acquired-pneumonia

\begin{tabular}{llc}
\hline Risk Factors & OR $(\mathbf{9 5} \% \mathbf{C I})$ & $\mathbf{p}$ \\
\hline Age & $1.03(1.01-1.05)$ & 0.002 \\
Antacids & $5.29(1.89-14.79)$ & 0.001 \\
CNS Disease & $3.13(1.24-7.93)$ & 0.02 \\
\hline
\end{tabular}

CI, confidence interval; CNS, central nervous system; OR, odds ratio.

Table 3. Microorganisms recovered from patients with nonventilator associated hospital acquired-pneumonia

\begin{tabular}{lccc}
\hline Microorganisms & Cases & $\begin{array}{c}\text { Specimens } \\
\text { Blood cultures }\end{array}$ & BAL \\
\hline MSSA & 2 & 1 & 1 \\
MRSA & 2 & 2 & 0 \\
Proteus ssp. $^{\text {a }}$ & 2 & 0 & 2 \\
P. aeruginosaa & 1 & 0 & 1 \\
Total & 7 & 3 & 4 \\
\hline
\end{tabular}

BAL, Bronchoalveolar lavage; MSSA, Methicillin-Susceptible Staphylococcus aureus; MRSA, Methicillin-Resistant Staphylococcus aureus. ${ }^{a}$ All Gram-negative isolates were susceptible to most Quinolones, Aminoglicosides, 3rd/4th generation Cephalosporins and Carbapenems.

of the lower airways $[14,18]$. However, the importance of the "gastropulmonary route" for colonization and infection of the respiratory tract has been challenged. Recent research suggests a predominant role for oropharyngeal colonization and micro-aspiration of microorganisms in the pathogenesis of hospital-acquired pneumonia [19]. Still, studies that favor either gastric or oropharyngeal colonization as a source of pathogens for hospital-acquired pneumonia enrolled patients under mechanical ventilation $[18,19]$. We can only hypothesize that, among nonventilated patients, contaminated gastric content could reach lower airways through reflux.

The pathogenesis of VAP and NVHAP may differ in several ways. Feldman et al. documented the rapid development colonization in the oropharynx, stomach, lower airways and endotracheal tube in patients under mechanical ventilation [20]. The dynamics of colonization is dependent on the extensive and aggressive manipulations to which those patients are exposed. This is not the case for nonventilated patients. In this group, changes in oropharyngeal flora may be delayed. Etiological aspects of NVHAP may resemble those from community-acquired or early-onset hospital-acquired pneumonia [15,21]. Our findings agree with this hypothesis.

Patients from our study were not submitted to extensive etiological investigation. However, among the few subjects with valid microbiological results, most had Methicillinsusceptible Staphylococcus aureus or multidrug-susceptible Gram-negative bacilli.

Most studies that address NVHAP focus on patients with special predisposing conditions, such as surgical procedures or immunossupression $[1,6]$. Few authors selected cases and controls according to criteria similar to those used in our study.
Barreiro-Lopez et al. analyzed data from 67 case patients and 67 controls matched by age, sex and period of admission [21]. Those authors found previous surgery, bronchoaspiration and the use of Steroids as significant risk factors for NVHAP. A cohort study by Nicolas Sanchez et al. identified several predictors of NVHAP: use of Sedatives, Calcium Channel Blockers and Amoxicillin-Clavulanate; placement of Nasogastric tubes; and elevated serum Creatinine levels [22].

Those findings differ from ours. Few case patients in our study had undergone surgery. Naso-enteral tube feeding was a significant risk factor in univariate analysis, but not in the multivariable model. Finally, the previous use of antimicrobials did not increase NHVAP incidence - though it may have contributed to the finding of many negative blood and BAL cultures.

Since NVHAP cases still occur, it is important to understand its epidemiology and find out opportunities for preventive strategies. The finding of age and Central Nervous System diseases as risk factors points out to groups that require special attention from the infection control team. Also, our findings suggest that a more rational use of Antacids may help in the prevention of pneumonia. From our perspective, NVHAP may be an old issue, but also one that should be addressed in the light of recent knowledge.

\section{References}

1. Agusti C, Rano A, Sibila O, Torres A. Nosocomial pneumonia in immunosuppressed patients. Infect Dis Clin North Am, 2003; 17:785-800.

2. Sopena N, Sabria M. Neumonía nosocomial en el paciente no ventilado. Enferm Infecc Microbiol Clin 2006; 23 (suppl 3): 24-9.

3. Hanson LC, Weber DJ, Rutala WA. Risk factors for nosocomial pneumonia in the elderly. Am J Med 1992; 92: 161-166.

4. Loeb M, McGeer A, MacArthur M, Walter S, Simor AE. Risk factors for pneumonia and other lower respiratory tract infections in elderly residents of long-term care facilities. Arch Intern Med 1999; 159: 2058-2064.

5. Hooton TM, Haley RW, Culver DH, White JW, Morgan WM, Caroll RJ. The joint associations of multiple risk factors with the occurrence of nosocomial infection. Am J Med 1981; 70: 960-970.

6. Windsor JA, Hill GL. Risk factor for postoperative pneumonia. The importance of protein depletion. Ann Surg 1988; 208: 209-214

7. Centers for Disease Control and Prevention. NNIS criteria for determining nosocomial pneumonia. Atlanta, GA: U.S. Department of Health and Human Services, CDC, 2003.

8. World Health Organization. International Classification of Diseases. tenth revision, 2005 update. Geneva, Switzerland: WHO; 2005.

9. Charlson ME, Pompei P, Ales KL, MacKenzie CR. A new method of classifying prognostic comorbidity in longitudinal studies: development and validation. J Chronic Dis 1987; 40: 373-383.

10. Collins TC, Daley J, Henderson WH, Khuri SF. Risk factors for prolonged length of stay after major elective surgery. Ann Surg 1999; 230: 251-9.

11. NCCLS. Performance standards for Antimicrobial Disk Susceptibility Tests; Approved Standard-Eighth Edition. NCCLS document M2-A8. Pennsylvania: NCCLS, 2003.

12. Celis R, Torres A, Gatell JM, Almela M, Rodríguez-Roisin R, AgustíVidal A. Nosocomial pneumonia. A multivariate analysis of risk and prognosis. Chest 1988; 93: 318-324. 
13. Bontem MJM, Kollef MH, Hall JB. Risk factors for ventilatorassociated pneumonia: from epidemiology to patient management. Clin Infect Dis 2004; 38: 1141-1149.

14. Centers for Disease Control and Prevention. Guidelines for preventing Health-care-associated pneumonia, 2003. MMWR 2004; 53 (RR-3): 1-35.

15. Sopena N, Sabrià M, The Neunos 2000 Study Group. Multicenter study of hospital-acquired pneumonia in non-ICU patients. Chest 2005; 127: 213-19.

16. Feldman C. Pneumonia in the elderly. Clin Chest Med 1999; 20: 563-73.

17. Hilker R, Poetter C, Findeisen N, Sobesky J, Jacobs A, Neveling M, Heiss WD. Nosocomial pneumonia after acute stroke: implications for neurological intensive care medicine. Stroke 2003; 34: 975-81.

18. Torres A, El-Ebiary M, Soler N, Monton C, Fabregas N, Hernandez C. Stomach as a source of colonization of the respiratory tract during mechanical ventilation: association with ventilatorassociated pneumonia. Eur Respir J 1996; 9: 1729-35.
19. Bontem MJM, Kollef MH, Hall JB. Risk factors for ventilator-associated pneumonia: from epidemiology to patient management. Clin Infect Dis 2004; 38: 11411149.

20. Feldman C, Kassel M, Cantrell J, Kaka S, Morar R, Goolam Mahmed A, Phillips JI. The presence and sequence of endotracheal tube colonization in patients undergoing mechanical ventilation. Eur Respir J 1999; 13: 54651 .

21. Barreiro-Lopez B, Tricas JM, Mauri E, Quintana S, Garau J. Factores de riesgo y pronósticos de la neumonía nosocomial en los pacientes no ingresados en unidades de cuidados intensivos. Enferm Infecc Microbiol Clin. 2005; 23: 51924.

22. Nicolas Sanchez FJ, Vila Justribo M, Rubio Caballero M. Factores de riesgo de la neumonía nosocomial fuera de la UCI. An Med Interna 2000;17:247-53. 